\title{
MÁS ALLÁ DE LA DISCIPLINA ACADÉMICA: ALGUNAS CONSIDERACIONES PARA EL DESARROLLO EN LOS PROCESOS EDUCATIVOS
}

\author{
BEYOND ACADEMIC DISCIPLINE: SOME CONDITIONS \\ FOR THE DEVELOPMENT OF THE TEACHING PROFESSION
}

\author{
Sandra Patricia Roncancio Sastoque ${ }^{a}$, Óscar Mauricio Penagos Rojas ${ }^{a^{*}}$ \\ y José González Such ${ }^{b}$
}

Fechas de recepción y aceptación: 13 de julio de 2019, 27 de enero de 2020

DOI: $10.46583 /$ edetania_2020.57.471

Resumen: Erróneamente se cree que enseñar es una actividad sencilla en la que solo basta con conocer la materia y algo de experiencia. Desconociendo los conocimientos pedagógicos y didácticos propios de la docencia y de otras áreas como la psicología cognitiva y la sociología, que facultan para ejercer de una manera profesional la enseñanza. Por otra parte, se desconoce la evolución en las prácticas y necesidades educativas del mundo contemporáneo que van más allá de un conocimiento disciplinar. En este trabajo se describen algunos factores a tener en cuenta para el desarrollo de la profesión docente, al existir una relación multidimensional (normativa, emocional, intersubjetiva), centrada en objetivos generales, ambiguos, heterogéneos e inmersa en seres humanos, que en la actualidad demandan del desarrollo de ciertas competencias como: alteridad y buen trato, motivación constante por aprender, trabajo en equipo y procesos de metacognición y autorregulación; las cuales han de ser promovidas y articuladas no solo desde la escuela, sino de manera conjunta en el entorno familiar y social.

Palabras clave: Actitud docente, motivación, trabajo en equipo, metacognición y autorregulación, formación docente.

a Estudiante de doctorado en Educación. Universidad de Valencia.

${ }^{\text {b }}$ Facultad de Filosofía y Ciencias de la Educación. Universidad de Valencia.

* Correspondencia: Óscar Mauricio Penagos Rojas. Diagonal 20A \# 10 - 66 código postal. 250251 Cundinamarca. Zipaquirá. Colombia.

E-mail: penagos06@gmail.com 
Abstract: It is mistakenly believed that teaching is a simple activity in which to know the subject matter and some experience is enough. Not knowing the pedagogical and didactic knowledge of teaching and other areas such as cognitive psychology and sociology, which empower to practice teaching in a professional manner. On the other hand, the evolution in the practices and educational needs of the contemporary world that go beyond disciplinary knowledge is unknown. This paper describes some factors that should be taken into account for the development of the teaching profession, as there is a multidimensional relationship (normative, emotional, intersubjective), focused on general, ambiguous, heterogeneous and immersed in human beings objectives, which currently demand the development of certain skills such as: otherness and good treatment, constant motivation to learn, teamwork and metacognition and self-regulation processes; which have to be promoted and articulated not only from school, but jointly in the family and social environment.

Keywords: Teaching attitude, motivation, teamwork, metacognition and self-regulation, teacher training.

\section{INTRODUCCIÓN}

Los enfoques educativos a lo largo del tiempo han cambiado de manera vertiginosa. En la educación tradicional la formación de los educandos se centraba en la instrucción, obediencia y mecanización de los conocimientos, donde difícilmente se formaba en otros aspectos o necesidades sociales; actualmente dichas necesidades sociales han evolucionado a una multiplicidad de formas más complejas que la escuela debe contemplar. Para esto las instituciones educativas han de estar inmersas en el reconocimiento, caracterización y en la manera de suplir los intereses de sus estudiantes, y esto requiere del esfuerzo conjunto de manera organizada de toda una comunidad.

Dentro de estas necesidades sociales que hay que considerar en el contexto actual tenemos la capacidad de saber interactuar con los demás de manera estratégica. Teniendo en cuenta que estas pueden ser de diferentes culturas, disciplinas, formas de pensar y actuar en un mundo globalizado, donde a cada minuto se desarrollan avances en múltiples saberes, de lo que resulta imposible a un solo individuo dominar tal cumulo de información. Por lo tanto, trabajar de forma conjunta y estar a la vanguardia es fundamental para desenvolverse de manera idónea tanto social, cultural, laboral como profesionalmente.

En este sentido, se pretende describir de manera sucinta algunos aspectos generales que se han de priorizar en los procesos de enseñanza y aprendizaje, 
como son: alteridad y buen trato, motivación, trabajo en equipo, procesos de metacognición y autorregulación académica y la formación continuada de los docentes (ver figura 1).

Por lo tanto, una condición para el desarrollo de estas necesidades ha de ser el priorizar su comprensión y puesta en práctica por parte de quienes se encuentran a cargo del direccionamiento y acompañamiento del desarrollo educativo de los estudiantes. Esto se debe a que los procesos educativos se desarrollan con mayor fluidez dentro de la convivencia cotidiana con el otro, hasta hacerlos parte de la cultura, lo cual refleja el nivel educativo alcanzado por una comunidad.

\section{FIGURA 1}

Condiciones básicas para la enseñanza y aprendizaje

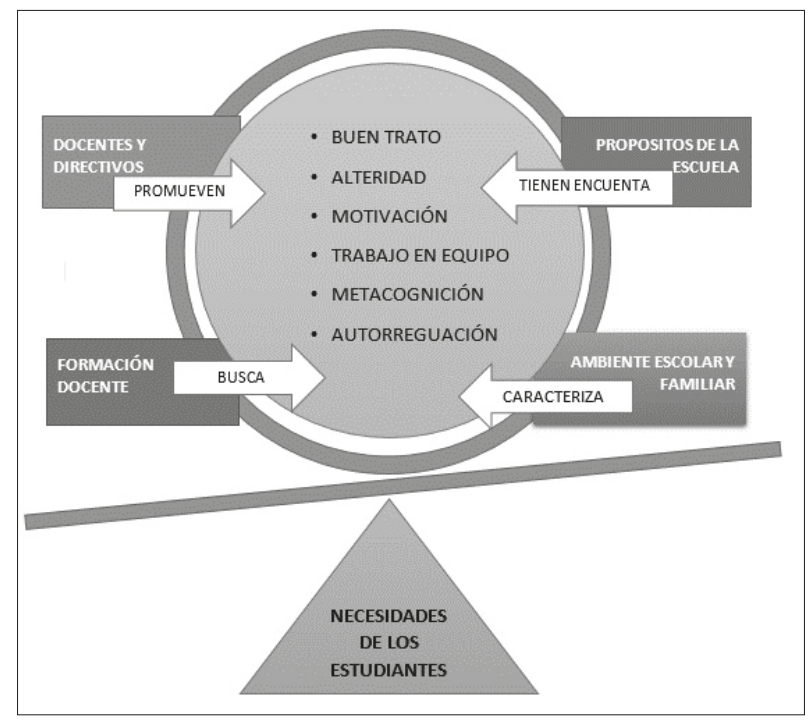

Fuente: Elaboración propia.

Así, las necesidades del estudiante (alteridad, motivación, trabajo en equipo y procesos de metacognición y autorregulación) deben ser tenidas en cuenta dentro de los propósitos de la escuela, promovidas por los docentes y directivos, quienes deben ser formados en estas para hacerlas parte de la cultura escolar y familiar. 


\subsection{Alteridad y buen trato}

Encontrarse el docente en un aula colmada de diversas personalidades, dispuestas a escuchar o no escuchar es un desafío que enfrenta cada día. La pregunta es cómo hacer para que los estudiantes no se cohíban en preguntar o que se les permita plantear sus dudas, ideas, opiniones de una manera organizada: ¿qué es esto?, ¿no entiendo?, ¿por qué es así?, y que muestren interés y desvirtúen el "no puedo", pues la relación educativa entre docente y estudiante influye en el rendimiento académico, en la actitud hacia la materia y en el desarrollo personal del estudiante, puesto que si partimos de un ambiente de escucha, tolerancia, alteridad, confianza y empatía el discente dará su mejor esfuerzo, el docente lo reconocerá y lo guiará a que alcance el desempeño deseado y el "no puedo" desaparezca de su mente.

Toda institución educativa se debe caracterizar por la vivencia de valores fundamentales como por ejemplo el respeto, la honradez, la tolerancia, la alteridad y otras. Cualidades de vital importancia entre todos los miembros de una comunidad educativa y especialmente docentes y alumnos. Este conjunto de valores contribuye en la consolidación de actitudes democráticas al dar oportunidad a través de la comunicación asertiva de contrastar y construir diversas formas de pensamiento.

Se debe dialogar con amor dejando de lado la pasividad, el desinterés y la indiferencia. Si aplicamos el valor de la alteridad al escuchar, argumentar, orientar y acordar, brindamos al error o equivocación la oportunidad de promover aprendizajes más profundos concibiendo el acto de enseñar como un acto de humildad y tolerancia colocándonos en la posición del otro, aceptando sus errores y corrigiéndolos con amor y respeto. Por lo tanto, los centros educativos se han de caracterizar por un ambiente de sana convivencia en donde se piense y trate de entender al otro en cada momento, donde se permita brindar opiniones frente a gustos y desavinieres, suscitar espacios de discusión, así como de relajación y reflexión, colmados de diversidad donde todos aprendemos.

La valoración de los progresos más allá de los resultados, la comprensión de los errores, la confianza depositada en las capacidades de sus estudiantes, la manera de explicar y propiciar la participación y la cooperación en el aula parten de la buena actitud del docente (Núñez del Rio, 2009). Entusiasmado 
por enseñar, vocación por sentir y ver el avance de su estudiante, disposición a dar lo mejor de sí mismos, esfuerzo, dedicación y amor a su labor con el propósito de que su discípulo comprenda y llegue más allá del contenido a un proceso cognitivo que lo posesione en una sociedad del conocimiento. Todas estas características se reflejan cuando el docente busca interactuar con los estudiantes no solo dentro del aula, sino creando lazos de confianza y amistad, al conversar para conocer sus inquietudes, expectativas, aspiraciones, problemas de tal modo que el estudiante se identifique con su profesor y despierte el interés por el aprendizaje.

Así, los educandos son individuos únicos que el docente debe tomarse el tiempo de conocer. García (2014) refiere que ellos son personas con iniciativa, con necesidades personales de crecer, con potencialidad para desarrollar actividades; son seres que participan no solo cognitivamente, sino personas con afectos, inquietudes, intereses, valores e ideas particulares. El docente debe tomarlos en cuenta con sentimientos de afecto, alteridad y consideración, ya que son seres que requieren mucha atención, compromiso, agrado y cordialidad; muchas veces su familia está ausente y el ejemplo de adulto que seguir son los docentes que permanecen con ellos entre 6 a 8 horas al día durante cinco días a la semana.

Como es sabido, los procesos educativos son propios de una acción social, donde la interacción con los demás es esencial para el cumplimiento de este fin. Por tal motivo estas relaciones sociales han de ser de total agrado para cada uno de los integrantes de una sociedad educativa, ya que asistir a un lugar donde percibimos cierta incomodidad o donde se nos tenga en cuenta solamente para una necesidad académica, desconociendo otros aspectos de gran importancia como el desarrollo social y afectivo, afecta a la motivación por aprender. De ahí que la escuela debería tener el compromiso de promover experiencias o situaciones en las que cada uno de sus integrantes y en especial los estudiantes conciban relaciones más profundas mediante la práctica de la solidaridad, el respeto, la compasión, el perdón, la alteridad y aquellas que se aprecien como carentes en el entorno, ya que todas estas son fundamentales entre individuos que comparten a diario y más aún en procesos educativos en los cuales se busca formar a personas íntegras que forjarán una mejor sociedad.

A su vez, las relaciones sociales en la escuela se deben caracterizar por una gran carga de experiencias positivas. Vivencias emotivas a través del ejemplo 
entre docentes para que los estudiantes se apropien de dichas conductas de gran valor emocional, proporcionando actitudes y valores necesarios en los procesos de aprendizaje. Por lo cual los educadores se han de capacitar en formas de adquirir y desarrollar un alto grado de inteligencia emocional que les permita el manejo adecuado de diversas situaciones y necesidades de sus educandos. Esto solo puede forjarse cuando el docente se entusiasma con su labor y la observa como un reto, al percibir la vida desde una perspectiva diferente a la propia y compartiendo emociones con quienes lo rodean con el firme propósito de contribuir en la formación de toda la comunidad y a su vez generando un espacio para continuar aprendiendo.

\subsection{Motivación}

Es un hecho que la motivación influye en el aprendizaje. Esta se refiere a los propósitos que dan sentido, energía y dirección al actuar, su estudio nos permite conocer por qué y para qué se inicia una conducta, se mantiene en el tiempo y se detiene, por lo que el maestro, los padres de familia y todos los responsables en la formación de los niños y adolescentes necesitan incitarlos a fijarse objetivos claros y específicos, los cuales orienten sus emociones y acciones debido a que las personas que no se proponen metas difícilmente avanzan en su desarrollo personal y académico para obtener resultados constructivos y con sentido para sus vidas. Así que enriquecer al estudiante con palabras de autoestima, autocontrol y autorreflexión en cada clase es primordial; comunicarle de manera clara el para qué de lo que está aprendiendo le va ayudar a interesarse por comprender de forma consciente más que en aprobar, y de ese modo podrá ser una persona exitosa a nivel emocional, social, familiar y laboral.

Para lograr llevar a cabo lo mencionado anteriormente debemos tener en cuenta las necesidades y algunas condiciones que la psicología ha llegado a plantear en la búsqueda del sano desarrollo y el bienestar de los individuos. Diferentes estudios, entre los que destacan la teoría de la autodeterminación (TAD), apoyada por la teoría de la evaluación cognitiva (TEC), y la teoría de la integración organísmica (TIO) (Ryan y Deci, 2000), el componente "sensación de flujo o reto óptimo" (Csikszentmihalyi, 2004) y la influencia de las 
recompensas externas (Mares, 2009) nos han permitido avanzar en la comprensión de algunos principios que se deben tener en cuenta para ayudar a nuestros estudiantes a desarrollar una motivación genuina por aprender.

La teoría de la autodeterminación planteada por Ryany Deci (2000) es un enfoque hacia el desarrollo de la motivación humana y la personalidad. Se preocupa por establecer las necesidades psicológicas básicas de los individuos, los factores que intervienen en la autorregulación de la conducta propuesta en la TEC y la manera en la cual diferentes tipos de motivación y contextos pueden impedir o promover la internalización o integración de la regulación de la conducta, explicado desde la TIO.

Dentro de las necesidades psicológicas básicas propuestas tenemos la de ser competente, relacionarse y la autonomía. Estas son innatas, esenciales y han de ser satisfechas de manera continua durante el transcurso de toda la vida para que el individuo experimente un sentido de integridad y bienestar. La necesidad de relacionarse se presenta cuando el individuo se siente vinculado, aceptado, apreciado, querido y respetado por otras personas de su interés o con quienes comparte a diario y la autonomía es la necesidad de manejar la propia conducta sin que exista algún tipo de control externo. Cuando estas necesidades psicológicas básicas se encuentran satisfechas se promueve la motivación.

La necesidad de competencia hace referencia a la "sensación o creencia" de idoneidad o efectividad. Se desarrolla al interactuar con el entorno y descubrir que esta interacción produce un hecho o resultado, llevándonos a pensar "yo puedo", "lo logré" o "lo hice", elevando nuestra motivación con el paso del tiempo y las experiencias vividas. Esta percepción puede variar en el caso de situaciones no favorables, donde el efecto se puede revertir mediante la orientación oportuna y pertinente del docente.

Asimismo, la TEC determina que la percepción de competencia no es suficiente para promover conductas autodeterminadas. Esta ha de ser acompañada por un sentido de autonomía que permita sentir la tarea como propia y no como algo impuesto. En donde la introducción de recompensas o castigos externos altera el locus o el origen de la motivación hacia el desarrollo de la actividad, reduciendo su interés por la tarea en sí y transfiriendo su atención en la recompensa o el castigo a recibir, disminuyendo la autonomía del individuo. De acuerdo con Amabile (1996), Grolnick y Ryan (1987) y Utman 
(1997, como se citó en Ryan y Deci, 2000) los estudiantes que son enseñados con un enfoque controlador muestran una pérdida de iniciativa y reducción en el aprendizaje, especialmente en lo conceptual y creativo.

Además, la TIO establece que se puede pasar de una conducta no autodeterminada a una autodeterminada a través de la integración e internalización de los motivos. Estos llegan al individuo de manera externa y se van transformando mediante una serie de procesos que le permiten otorgarle sentido, al comprender el para qué de aquello que hace y encontrar el gusto mientras lo hace propio. Dichas etapas se pueden apreciar en la tabla 1.

\section{TABLA 1}

El continuo de la autodeterminación que muestra los tipos de motivación con sus estilos regulatorios, el locus de causalidad y los procesos correspondientes (tomado de Ryan y Deci, 2000)

\begin{tabular}{|c|c|c|c|c|c|c|}
\hline \multirow{3}{*}{$\begin{array}{l}\text { Conducta } \\
\text { Motivación } \\
\begin{array}{l}\text { Estilos regu- } \\
\text { latorios }\end{array}\end{array}$} & \multicolumn{5}{|c|}{ No autodeterminada } & Autodeterminada \\
\hline & Desmotivada & \multicolumn{4}{|c|}{ Motivación extrínseca } & $\begin{array}{l}\text { Motivación } \\
\text { intrínseca }\end{array}$ \\
\hline & No regulación & $\begin{array}{l}\text { Regulación } \\
\text { externa }\end{array}$ & $\begin{array}{l}\text { Regulación } \\
\text { introyectada }\end{array}$ & $\begin{array}{l}\text { Regulación } \\
\text { identificada }\end{array}$ & $\begin{array}{l}\text { Regulación } \\
\text { integrada }\end{array}$ & Intrínseca \\
\hline $\begin{array}{l}\text { Locus de } \\
\text { causalidad } \\
\text { percibido }\end{array}$ & Impersonal & Externo & Algo externo & Algo interno & Interno & Interno \\
\hline $\begin{array}{l}\text { Procesos } \\
\text { regulatorios } \\
\text { relevantes }\end{array}$ & $\begin{array}{l}\text { No intencional } \\
\text { No evaluativo } \\
\text { Incompetencia } \\
\text { Falta de con- } \\
\text { trol }\end{array}$ & $\begin{array}{l}\text { Obediencia } \\
\text { Recompensas } \\
\text { externas y } \\
\text { castigos }\end{array}$ & $\begin{array}{l}\text { Autocontrol } \\
\text { Egoimplica- } \\
\text { ción } \\
\text { Recompensas } \\
\text { internas y } \\
\text { castigos }\end{array}$ & $\begin{array}{l}\text { Importancia } \\
\text { personal } \\
\text { Valor cons- } \\
\text { ciente }\end{array}$ & $\begin{array}{l}\text { Congruencia } \\
\text { Consciencia } \\
\text { Síntesis con } \\
\text { el yo }\end{array}$ & $\begin{array}{l}\text { Interés } \\
\text { Gozo } \\
\text { Satisfacción } \\
\text { inherente }\end{array}$ \\
\hline
\end{tabular}

Por otra parte, Csikszentmihalyi (2004) propone su teoría del flujo o reto óptimo. Dicha teoría se describe como un estado mental en el cual la persona se encuentra focalizado, completamente comprometido e inmerso en una actividad en la que tendrá éxito. Donde esta experiencia se caracteriza por objetivos y normas claras, alcanzables acordes al conjunto de habilidades y destrezas de quien realiza la tarea; concentración y enfoque, relación con una única actividad al profundizar en el asunto; retroalimentación directa e inmediata, pues las equivocaciones o fallos en el transcurso de los procesos 
de aprendizaje son obviamente esperados, y por lo tanto un acompañamiento y correcta orientación marcan la diferencia para ajustar el comportamiento; equilibrio entre el nivel de habilidad y el desafío, dado que la actividad no debe ser ni demasiado fácil ni muy complicada, sino que ha de ir evolucionando hasta alcanzar la proficiencia en aquello que deseamos enseñar, pues la tarea es intrínsecamente gratificante cuando no se nota el esfuerzo; pérdida o distorsión del sentido del tiempo (se percibe que transcurrieron minutos cuando en realidad pasaron horas en el desarrollo de la actividad), y un sentimiento de control personal de la situación o actividad. Según el autor no todos los componentes son necesarios para conseguir una experiencia de flujo (ver figura 2).

FIGURA 2

Experiencia de flujo (tomado y adaptado de Csikszentmihalyi, 2004)

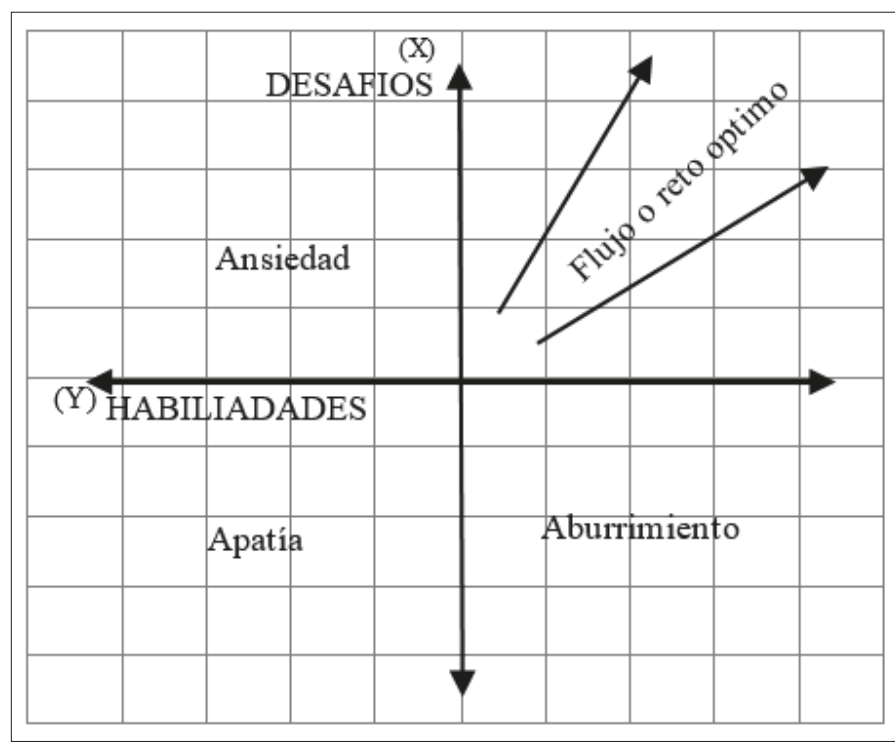

Es así como el hecho de conocer, implementar y fortalecer estas y otras teorías sobre la motivación nos permite a los docentes plantear una perspectiva diferente a nuestros estudiantes. Asistir a la escuela debe ser percibido como una experiencia de goce y disfrute por aprender y no una imposición 
o castigo. Así, los contextos caracterizados por la autonomía, la competencia y el relacionarse o vincularse socialmente fomentan una mayor internalización e integración de la motivación, a diferencia de los contextos que frustran satisfacer estas necesidades por medio del control excesivo, los desafíos inapropiados, muy fáciles o muy complicados, y la falta de relacionarse con otros promueven el desinterés, la irresponsabilidad y un ambiente de estrés, cohibiendo el desarrollo de la motivación y al mismo tiempo limitando los niveles del aprendizaje.

\subsection{Procesos de metacognición y autorregulación académica}

El desarrollo de las competencias en el estudiante se rige por el principio educativo de aprender a aprender. Es interiorizado y adoptado por este al comprometerse con su propio aprendizaje para lograr el éxito en su formación. Estos educandos son proactivos debido a que realizan los procesos de metacognición y autorregulación, los cuales son acciones que se complementan y donde el primero reflexiona sobre sus propios conocimientos para mejorarlos y el segundo de forma autónoma y consciente traza un plan estratégico para superar las falencias encontradas al planear, supervisar y evaluar dicha estrategia de manera constante. En otras palabras, la unión de estos procesos promueve la búsqueda de información cuando la necesitan sin que el profesor lo pida, y cuando los recursos son escasos en sus familias encuentran la manera de solucionarlo preguntando a su maestro o pares por conocimientos que no comprenden; planean, agrupan metas, organizan, se automonitorean y se autoevalúan en el momento que están aprendiendo algo.

Por tanto estos aprendices tienen un alto grado de autoestima, autoeficacia, motivación y creen en sus propias capacidades para asegurar el éxito en sus tareas. Ellos buscan consejo e instrucción, y reconocen si son principiantes en determinado tema, sin considerar que lo saben todo. Su comportamiento se encamina a la selección, estructuración y creación de ambientes donde pueden aprender, con el fin de reforzar lo que ya saben, es decir, incitan su propia instrucción (Zimmerman, 2011). El resultado de su desempeño es monitoreado por ellos mismos para alcanzar los objetivos deseados, buscando estrategias que los ayuden a mejorar si es el caso. 
En este orden de ideas, la autorregulación ayuda al estudiante a ser más activo y constructivo, lo incitan a monitorear y regular su pensamiento, motivación y comportamiento, vinculando estrategias cognitivas, metacognitivas y motivacionales. De manera más puntual, las estrategias de cognición incluyen habilidades para codificar, relacionar, jerarquizar, memorizar y recuperar información, lo que conduce a procesos más complejos de elaboración, organización y resolución de problemas en el aprendizaje de una materia, mientras que las de metacognición son secundarias, se refieren a la planificación, regulación, observación y modificación de los propios procesos cognitivos. Por tanto, las estrategias cognitivas actúan sobre un problema o tarea específica con el objeto de facilitar su adquisición y las metacognitivas reflexionan sobre lo aplicado, el pensamiento y lo aprendido, si quedó reforzado, donde las estrategias motivacionales juegan un papel incluyente de creencias y actitudes del discente para el desarrollo de las dos primeras estrategias. Todo este proceso se ejerce con la ayuda del maestro y sus habilidades para que el estudiante se encuentre con sus intereses y el conocimiento.

De esto se infiere que conocer y comprender cómo los estudiantes pueden regular su proceso de aprendizaje permite que los docentes sean parte de esta iniciativa con diversas estrategias metodológicas, llevando lo aprendido a diversos contextos para construir conocimiento de forma significativa, y comenzando con reflexiones intrínsecas al propio docente, como: ¿qué tanto contribuimos a desarrollar en el estudiantado habilidades y actitudes para el aprendizaje continuo?, desde nuestra propia práctica como docentes, ¿cómo aportamos a dichos aprendizajes?, ¿en qué medida siendo docentes hemos desarrollado habilidades y actitudes para nuestro propio aprendizaje continuo, promoviendo una cultura académica orientada a revitalizar nuestro quehacer? Y en este sentido orientaremos preguntas para elaborar rúbricas de autoevaluación de los procesos cognitivos, metacognitivos, motivacionales y conductuales del estudiante, como por ejemplo: ¿comprendí en su totalidad el tema?, ¿qué voy hacer en mi casa para poder comprender la temática de mejor manera?, ¿qué tengo que hacer para poder aprender este tema?, ¿qué conocimientos previos debo saber para aprender mejor este tema?, ¿quiero aprender este tema?, ¿para qué me sirve aprender este tema? y ¿me cuesta trabajo esto?, ¿es un reto para mí lograrlo? Así, los estudiantes con este valor 
añadido realizan sus tareas y proyectos con confianza, diligencia y recursividad; ellos saben y reconocen tanto sus habilidades como las que no poseen.

\subsection{Trabajo en equipo como competencia que desarrollar}

Trabajar en equipo es una competencia bastante compleja, difícil de alcanzar por la demanda de diferentes conocimientos, habilidades, actitudes y aptitudes que por lo general varían de acuerdo con el contexto y la tarea que hay que desarrollar (Torrelles et al., 2011). La consolidación de equipos de trabajo se convierte en algo fundamental, ya que aporta más beneficios a las diferentes organizaciones (familiares, sociales, educativas, empresariales) que el trabajo de manera individual. Donde los docentes tenemos la oportunidad y el deber de promover de manera consciente en nuestros estudiantes el desarrollo de dicha competencia, dada su importancia para convivir y obtener grandes resultados.

Tradicionalmente la formación en la escuela se basa en la transmisión de contenidos. Los docentes en muchas situaciones utilizan el trabajo en equipo como estrategia metodológica con sus estudiantes, pero en la mayoría de los casos no se detienen a analizar la manera en que se desenvolvieron dentro de este, de tal forma que les permita avanzar gradualmente hasta hacerlos competentes para trabajar en equipo. Enseñar dicha competencia es una tarea compleja que involucra tiempo y esfuerzo, ya que los conocimientos, habilidades y destrezas no son innatas en el ser humano, las cuales se deben propiciar, incentivar y desarrollar desde edades tempranas a través de la escuela junto con el entorno familiar y social.

Lo mencionado anteriormente se debe en parte a la falta de capacitación docente de manera continua. En este caso se debe conocer los diferentes modelos relacionados con los factores, condiciones, procesos y resultados, entre otros, del trabajo en equipo que le permitan implementar al maestro esta competencia dentro de su estructura de trabajo y entre docentes, para optimizar los procesos de enseñanza y aprendizaje al mismo tiempo que lo hace con sus estudiantes, ya que las visiones, perspectivas y enfoques que surgen como necesidades sociales a lo largo del tiempo exigen estar a la vanguardia de este fenómeno. 
Es cierto que el docente con otros docentes realiza un trabajo en equipo parcial. Se elaboran, articulan y ajustan proyectos transversales, actividades pedagógicas, planes de área y asignatura, entre otras al comienzo del año lectivo, pero se hace necesario brindar espacios y tiempos para dar seguimiento y control a su desarrollo, lo cual se debe programar de manera periódica, enriqueciendo y fortaleciendo estas y el trabajo conjunto, dejando de lado el espíritu individualista que por tradición ha regido al docente desde hace mucho tiempo. La educación es una de las últimas profesiones donde todavía es legítimo trabajar solo en un espacio a salvo de invasores (Rudduck, 1991). La labor individual priva al docente de una retroalimentación significativa, puesto que las complejas dinámicas de los centros educativos exigen un funcionamiento integrado, articulado y conjunto entre el profesorado que fomenten la reflexión diaria entre compañeros sobre su práctica docente, propiciando el crecimiento en cada uno de sus estudiantes y de ellos mismos.

Actualmente se ha aceptado por la comunidad científica modelos que explican la incidencia de diferentes factores y los procesos que intervienen en el desarrollo de la competencia de trabajo en equipo. El modelo integrado de efectividad en equipo, desarrollado en los años 2005 y 2008 por Ilegen, Hollenbeck, Johnson y Jundt, y Mathieu, Maynard y Rapp, adaptado al ámbito docente por Jaca, Viles y Zárraga-Rodriguez (2016) (ver figura 3), ha sido probado con datos experimentales en una escuela de ingenieros española y complementado con una herramienta propuesta por Jaca et al. (2016) que permite evaluar el funcionamiento de los equipos relacionado con el proceso operativo del modelo, permitiendo retroalimentar a los estudiantes.

Por otra parte, Torrelles et al. (2011) proponen una herramienta denominada Rúbrica de Teamwork (RUTE). Se ha utilizado para valorar la competencia de trabajo en equipo en estudiantes universitarios (Paris, Mas y Torrelles, 2016). Dicha herramienta se encuentra constituida por veintisiete dimensiones, diseñada con la finalidad de establecer el nivel adquirido sobre la competencia de trabajo en equipo. Donde cada una de las dimensiones cuenta con sus respectivas categorías, que las describen valoradas en una escala de uno a cuatro, indicando de menor a mayor dominio, permitiendo establecer un diagnóstico y así implementar acciones de mejora sobre el desarrollo de la competencia. Las dimensiones completas se pueden consultar en Paris et al. (2016). 
FIGURA 3

Adaptación del modelo IMO al ámbito docente (tomado de Jaca et al., 2016)

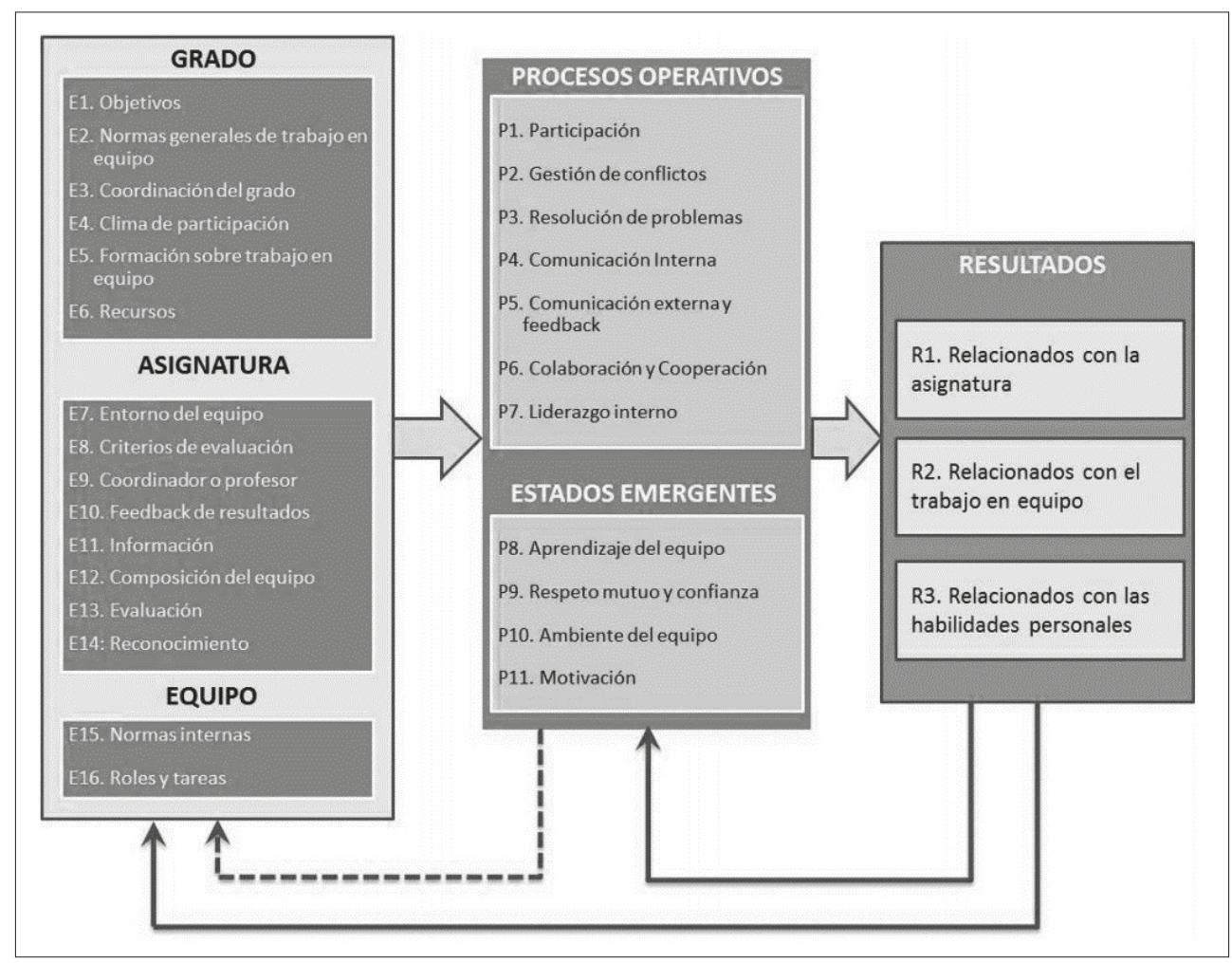

Asimismo, Solé, Casanellas, Collado, Pérez-Moreno y Sayós (2015 a,b,c) establecieron tres niveles de logro, con unos objetivos bien definidos para diferenciar cada uno de ellos, los cuales permiten determinar el progreso de los estudiantes en el dominio de la competencia como se muestra en la tabla 2. 
TABLA 2

Niveles de desarrollo de la competencia de trabajo en equipo

\begin{tabular}{|c|c|c|}
\hline Nivel & Resultados de aprendizaje & Evidencias para la evaluación \\
\hline Nivel 1 & $\begin{array}{l}\text { 1) Colaborar en el diseño de un planteamiento bási- } \\
\text { co de trabajo (cronológico) y participar en la discu- } \\
\text { sión de la estrategia de funcionamiento. } \\
\text { 2) Responsabilizarse de la realización de las tareas } \\
\text { individuales y del cumplimiento de los plazos. } \\
\text { 3) Aceptar que los objetivos comunes son priorita- } \\
\text { rios. } \\
\text { 4) Fomentar la confianza y la cordialidad en la } \\
\text { comunicación, así como la expresión del desacuerdo } \\
\text { sin tensiones. } \\
\text { 5) Valorar la aportación individual de cada miembro } \\
\text { del grupo de trabajo. } \\
\text { 6) Asumir el resultado del trabajo colectivo como } \\
\text { propio. }\end{array}$ & $\begin{array}{l}\text { Explicitar, mediante una reflexión indi- } \\
\text { vidual, qué han entendido los estudian- } \\
\text { tes que tienen que hacer y cómo se ha } \\
\text { planificado el trabajo. También cómo } \\
\text { se ha visto modificada su aportación a } \\
\text { partir de las intervenciones del resto de } \\
\text { los miembros. } \\
\text { Informe colectivo que explique el pro- } \\
\text { ceso de negociación grupal, en el cual } \\
\text { se plasmen las incidencias que se hayan } \\
\text { podido producir y las soluciones que se } \\
\text { han adoptado. } \\
\text { Cuestionario de evaluación individual } \\
\text { del proceso de trabajo en grupo. }\end{array}$ \\
\hline Nivel 2 & $\begin{array}{l}\text { 1) Fijar los objetivos del grupo y diseñar un plan de } \\
\text { trabajo realista y realizable. } \\
\text { 2) Distribuir las tareas y las responsabilidades, } \\
\text { identificando correctamente las individuales y las } \\
\text { colectivas. } \\
\text { 3) Intercambiar información, compartir recursos } \\
\text { personales, aportar ideas y modificar propuestas de } \\
\text { trabajo para fomentar la eficacia del grupo. } \\
\text { 4) Utilizar adecuadamente las habilidades comuni- } \\
\text { cativas y participativas, identificando quién aporta } \\
\text { qué respecto del objetivo grupal. } \\
\text { 5) Asumir la tarea individual como imprescindible } \\
\text { para lograr el resultado colectivo, que tiene que } \\
\text { poder ser defendido por cada uno de los componen- } \\
\text { tes del equipo. } \\
\text { 6) Realizar una evaluación ajustada del proceso de } \\
\text { trabajo y de los resultados conseguidos. }\end{array}$ & $\begin{array}{l}\text { Informe colectivo en el cual se plasme } \\
\text { la comprensión del grupo del trabajo } \\
\text { a realizar y la planificación que se ha } \\
\text { adoptado para llevarlo a cabo. } \\
\text { "Diario de campo" donde, individual- } \\
\text { mente, los miembros del grupo relaten } \\
\text { las vivencias en el seno del grupo y las } \\
\text { aportaciones que han ido realizando al } \\
\text { trabajo conjunto. } \\
\text { Informe colectivo que explique la fase } \\
\text { de revisión de los trabajos individuales } \\
\text { y el proceso de negociación que se ha } \\
\text { producido en el interior del equipo en el } \\
\text { momento de la puesta en común de las } \\
\text { tareas individuales. } \\
\text { Cuestionario de evaluación individual } \\
\text { del proceso de trabajo en grupo. }\end{array}$ \\
\hline
\end{tabular}




\begin{tabular}{|c|c|c|}
\hline Nivel & Resultados de aprendizaje & Evidencias para la evaluación \\
\hline Nivel 3 & $\begin{array}{l}\text { 1) Utilizar adecuadamente las habilidades colabora- } \\
\text { tivas, entre ellas el liderazgo compartido y rotatorio. } \\
\text { 2) Contribuir al reparto equilibrado de las activida- } \\
\text { des, asegurando la integración de los miembros y su } \\
\text { orientación hacia un rendimiento más elevado. } \\
\text { 3) Estimular y agradecer las aportaciones de los } \\
\text { otros, estimulando un clima interno de trabajo posi- } \\
\text { tivo y la cohesión del equipo. } \\
\text { 4) Negociar y resolver los problemas que surjan. } \\
\text { 5) Evaluar la efectividad del equipo, identificando } \\
\text { los factores que han contribuido a ella. } \\
\text { 6) Presentar los resultados del trabajo realizado. }\end{array}$ & $\begin{array}{l}\text { Reflexión colectiva continuada sobre: } \\
\text { - La tarea (definición, objetivos...). } \\
\text { - La organización (reparto de funciones } \\
\text { y responsabilidades, programación...). } \\
\text { - La negociación (conflictos, conoci- } \\
\text { mientos adquiridos, resultados a los que } \\
\text { se ha llegado...). } \\
\text { Autoevaluación individual de la aporta- } \\
\text { ción y la participación en el grupo. } \\
\text { Coevaluación del proceso y de los } \\
\text { resultados. }\end{array}$ \\
\hline
\end{tabular}

Fuente: Solé et al. (2015c).

Como podemos ver en estos ejemplos, la competencia trabajo en equipo ha alcanzado un desarrollo teórico bastante considerable, pretendiendo ir más allá de la instrumentalización como estrategia didáctica para llegar a ser un objetivo educativo, acorde con las necesidades que demanda la sociedad, pero poco o nada se trabaja con nuestros estudiantes y escasea con nuestros colegas.

\subsection{Formación del docente}

La formación continua en los docentes es una necesidad inherente de la profesión porque las sociedades son colectividades dinámicas. Se transforman y evolucionan, por ejemplo, en las relaciones y cuidados del medio ambiente; desarrollos teóricos y tecnológicos en múltiples áreas asociadas con la didáctica y pedagogía; formas de vida de los ciudadanos, relaciones entre padres e hijos y la manera de socializar con los demás; y la aceleración en el flujo de información, entre otras, hacen que se hable de nuevas percepciones y necesidades en la formación de los individuos, y a medida que estas se perciben se investigan, documentan e implementan, transformando las prácticas educativas de manera continua. 
El continuo cambio generacional de estudiantes que pasan por las aulas de los propios docentes, quienes desarrollan su profesión durante varias décadas, hace evidente una adaptación a los nuevos contextos sociales y culturales. Dicha formación continua permite el enriquecimiento de las estructuras conceptuales, llevándolo a un mejoramiento en la ejecución de su profesión. Se debe tener en cuenta conocimientos sobre el desarrollo de estrategias que fomenten la motivación por el aprendizaje, un ambiente escolar agradable, alteridad, metacognición, autorregulación y trabajo en equipo, necesidades que van más allá del paradigma de educar para la obediencia, la mecanización, repetición y la sumisión a un paradigma que busque brindar a nuestros estudiantes la oportunidad de interactuar y trabajar con los demás de manera respetuosa, crítica, argumentativa, creativa y propositiva como lo demanda la actual sociedad.

Las creencias, concepciones y orientaciones que posee un docente acerca de su quehacer influyen en el aula de clase. Básicamente depende del cómo se les enseñó, su proceso de formación inicial y experiencia laboral. Estos conocimientos se incrementan, transforman, asientan e interiorizan en las mentes de estos profesionales de manera implícita y explícita a medida que socializan, reflexionan y debaten con sus colegas en espacios de formación constante. Entre más el docente profundice e interiorice no sólo en su disciplina, sino en la complejidad de saberes que involucre la enseñanza podrá optimizar la selección, organización y articulación de aquello a enseñar, identificar problemas de aprendizaje en sus estudiantes, establecer estrategias de superación e incrementar la motivación y satisfacción profesional que se acrecienta a medida que mejora en su práctica al ver progreso en sus estudiantes mediante la innovación en los procesos educativos dentro y fuera del aula.

Dichos cambios son complejos y difícilmente modificables a menos que se realicen desde lo profundo de las estructuras conceptuales de los docentes. Solo se logra en la reflexión entre pares en una formación continua, dando como resultado procesos de enseñanza y aprendizaje más eficientes. Este proceso de reflexión busca analizar críticamente, revisar, construir y reconstruir su propia actuación en clase, lo cual es fundamental en relación con su quehacer diario, los propósitos de la disciplina que se ha de enseñar, la interacción con otros saberes, las actividades metodológicas que hay que implementar, como el uso de metáforas, analogías, representaciones y descripciones adaptadas al contexto, los materiales más convenientes para ser usados, las 
dificultades, concepciones, preconcepciones e ideas alternativas que poseen sus estudiantes, sexo y motivación, las estrategias y métodos de evaluación que beneficien su labor pedagógica y didáctica. Todo un bagaje de elementos que involucran los procesos de enseñanza y aprendizaje que demandan una reflexión constante para generar un cambio significativo.

Lo anteriormente mencionado es indispensable si consideramos la diversidad en los procesos de enseñanza y aprendizaje. A lo largo de su carrera los docentes siempre se van a encontrar con estudiantes con nuevas necesidades de aprendizaje, con nuevas formas de aprender, con condiciones y necesidades sociales diferentes cada vez más complejas y diversas, como por ejemplo pasar de una sociedad industrializada a una del conocimiento, de una operativa y jerarquizada a una de cooperación competente y descentralizada, de la pasividad y obediencia a una proactiva con iniciativa. Este cambio formativo se debe propiciar en la escuela, pero ¿cómo promover estos cambios sin que exista una formación continua de quienes tienen esta responsabilidad de enseñar?

La implementación exitosa de novedades educativas requiere que los profesores estén en constante aprendizaje para favorecer su trabajo. Los espacios de formación mediados por la reflexión del quehacer diario lleva a los docentes a realizar investigación sobre diferentes aspectos relacionados con los fines, las necesidades educativas, los procesos de enseñanza y aprendizaje y de evaluación, promoviendo acciones pedagógicas y didácticas innovadoras, eficaces, personalizadas y más eficientes, mejorando continuamente, lo cual debe ser fomentado en condiciones adecuadas y con el apoyo de entes externos que permitan a los maestros cumplir con una parte de su profesión, la de investigar generando conocimientos acordes a sus necesidades sin tener que estar permanentemente dependiendo de otros que les digan qué hacer, hasta llegar incluso a dificultar y/o entorpecer los procesos y fines propuestos, aunque sea con el ánimo de mejorar u ofrecer una educación de calidad. Han de ser los propios maestros quienes lideren sus propias investigaciones, lo cual les permitiría actuar con propiedad y de manera legítima, ya que la educación ha de ser comprendida, interiorizada por quienes la llevan a la práctica para que tenga sentido y deje de ser algo impuesto a la fuerza, mecanizado, sin llegar a comprender o dar sentido a lo que se hace. 
Por otra parte, el profesor debe ser el orientador o facilitador en la búsqueda de ese conocimiento práctico con sentido. Es decir, que la memorización de clases, las discusiones de personajes de historia y la lectura de libros en pro de identificar temas y caracteres no son bagajes suficientes de conocimiento; se debe pretender discutir múltiples interpretaciones de dichos textos con evidencia documental, enseñar a pensar con actitud investigativa. Por ello el docente requiere comprender el papel de la investigación como eje primordial para el aprendizaje de aquello que se estudia, transcendiendo algo más que un saber sin sentido. Es decir, usar el conocimiento en la realización de acciones, desempeños o productos flexibles para ser aplicados en situaciones diversas a aquellas en las que se aprendieron.

Lo anterior facilita promover habilidades de pensamiento interrelacionadas con las diferentes áreas, sin saturar y estresar a los estudiantes con gran cantidad de conocimientos enciclopédicos. Esto se podrá realizar mediante un trabajo articulado entre docentes de diferentes disciplinas. Por lo que es fundamental modificar los enfoques y procesos de enseñanza-aprendizaje para centrarlos en el desarrollo de habilidades de pensamiento, las cuales serán más significativas y útiles al prolongarse en el tiempo y servir de herramienta para continuar aprendiendo.

\section{Conclusiones}

Es difícil que los docentes o cualquier individuo domine todos los saberes y más aún cuando estos se transforman de manera tan vertiginosa en el contexto actual. Comprenderlos exige tiempo y esfuerzo, por lo cual se recomienda trabajar en equipo entre universidades que se encuentren a la vanguardia en el manejo de las necesidades, conocimientos, herramientas y demás que le competen a la escuela, para que actualicen y acompañen a los maestros dentro del aula a suplir dichas demandas.

Por otra parte, se debe dar mayor atención al quehacer diario "el vivir en la escuela", ya que esta vivencia es lo que en realidad forma a nuestros estudiantes. La experiencia del día a día en alteridad, respeto, trabajo en equipo, creatividad, resolución de problemas y actividades continuas donde se lleven 
a la práctica los contenidos disciplinares de manera articulada, no aislada o fragmentada ya que esto le quita sentido, utilidad e interés, desmotivando al estudiante para aprender.

De igual manera, los procesos educativos tienen estrecha relación en la forma que un individuo actúa en determinadas situaciones de acuerdo con sus conocimientos y experiencias. Así, la labor del docente requiere una formación específica, algo más que un saber académico debido a que se desenvuelve en medio de seres humanos conscientes y diversos, con contextos propios e independientes, únicos e irrepetibles, donde se toman decisiones que afectan a un grupo de personas. Por lo tanto, dichos procesos deben asumir el compromiso de alejarse de la mecanización u operativización y convertirse en algo único y amoldado a cada contexto.

Así, el estar en la escuela es un dispositivo formador de ciudadanos conectados con su propia experiencia, su propio mundo afectivo, cognitivo y comportamental y con el de los demás, con sus necesidades y con las del entorno; ciudadanos capaces de convivir de forma armoniosa, de construir nuevas formas de relación social basadas en el respeto y en las que la cooperación jugará un papel fundamental.

La profesión docente va más allá del trabajo directo en la escuela con los estudiantes, padres de familia y pares académicos. El educador requiere gozar de plena salud emocional y física como también de una formación continua en diferentes ámbitos, acorde a sus necesidades, las de sus estudiantes y el entorno laboral. Todo esto involucra y demanda recursos como tiempo, dinero y esfuerzo que se deben contemplar por parte de los dirigentes de la educación.

Asimismo, los fines y objetivos que se desarrollan en la escuela han de trascender esta, hasta llegar a ser un eje articulador en la familia y los diferentes entornos sociales. Movidos por los valores, el cuidado y la preservación del medio ambiente y el bienestar común, ya que el verdadero significado de la educación se debe entender desde la categoría de pensar y aplicar la alternativa de edificar un país mejor, crear un espíritu colectivo y creativo que estimule la colaboración entre individuos dispuestos a soñar ver un mundo diferente y capaz de lograr lo que se proponen por el bien de la humanidad. 


\section{BibliografíA}

Csikszentminalyi, M. (2004). TED Ideas worth spreading: flow, the secret to happiness. California, EE. UU.: TED. Recuperado de: <https://www.ted. com/talks/mihaly_csikszentmihalyi_on_flow>.

García-Rangel, G. (ed.), García, R., Karenina, A., Reyes, A. y Antonio, J. (2014). Relación maestro alumno y sus implicaciones en el aprendizaje. Ra Ximhai 10(5), 279-290.

Jaca, C., Viles, E. y Zárraga-Rodríguez, M. (2016). Desarrollo de la competencia de trabajo en equipo en un grado universitario. Memoria Investigaciones en Ingeniería (14), 23-34. Recuperado de: <https://www.researchgate.net/publication/311738931_Desarrollo_de_la_competencia_de_trabajo_en_equipo_en_un_grado_universitario $>$.

Mares, J. (ed.) (2009). Motivación y emoción. México, D. F.: Mc Graw Hill.

NúÑEz del Río, M. y Fontana, M. (2009). Competencia socioemocional en el aula: características del profesor que favorecen la motivación por el aprendizaje en alumnos de enseñanza obligatoria. REOP 20(3), 257-269.

Paris, G., Mas, O. y Torrelles, C. (2016). La evaluación de la competencia "trabajo en equipo" de los estudiantes universitarios. Revista d'Innovació Docent Universitària (8). Recuperado de: <https://www.researchgate.net/ publication/292320512_La_evaluacion_de_la_competencia_trabajo_en_ equipo_de_los_estudiantes_universitarios $>$.

RYAN, R. y DECI, E. (2000). La teoría de la autodeterminación y la facilitación de la motivación intrínseca, el desarrollo social y el bienestar. University of Rochester 55(1), 68-78. doi: 10.1037110003-066X.55.1.68.

RudDuck, J. (1991). Innovation and change. Reino Unido, Milton Keynes: Open University Press.

Solé, M. (coord.), Casanellas, M., Collado, A., Pérez-Moneo, M. y Sayós, R. (2015a). Rúbricas para la valoración del trabajo en equipo. Dipòsit Digital de la UB. Recuperado de: <http://diposit.ub.edu/dspace/handle/2445/63266>.

Solé, M. (coord.), Casanellas, M., Collado, A., Pérez-Moneo, M. y SAYós, R. (2015b). Trabajo en equipo. Indicaciones dirigidas al alumnado para adquirir la competencia en el grado de Gestión y Administración 
Pública. Dipòsit Digital de la UB. Recuperado de: <http:/hdl.handle. net $/ 2445 / 63268>$.

Solé, M. (coord.), Casanellas, M., Collado, A., Pérez-Moneo, M. y Sayós, R. (2015c). Trabajo en equipo. Indicaciones para el desarrollo y evaluación de la competencia genérica en el grado de Gestión y Administración Pública. Dipòsit Digital de la UB. Recuperado de: <http://hdl.handle. net/2445/63304>.

Torrelles, C., Coiduras, J., Isus, S., Carrera, F., París, G y Cela, J. (2011). Competencia de trabajo en equipo: definición y categorización. Profesorado. Revista de Currículum y Formación de Profesorado 15(3), 329-344. Recuperado de: <https://www.redalyc.org/pdf/567/56722230020.pdf $>$.

ZiMMERMAN, B. J. (2011). Motivational sources and outcomes of selfregulated learning and performance, en B. J. Zimmerman y D. H. Schunk (eds.), Handbook of self-regulation of learning and performance (pp. 49-64). Nueva York: Routledge. 